\title{
Human leukocyte antigen-DRB1 polymorphism in childhood acute lymphoblastic leukemia
}

\author{
MERVAT M. EL ANSARY ${ }^{1}$, LAMIAA A. MOHAMMED ${ }^{2}$, TAMER H. HASSAN ${ }^{3}$, \\ AHMED BARAKA ${ }^{2}$ and ALSHYMAA A. AHMED ${ }^{2}$ \\ ${ }^{1}$ Department of Clinical Pathology, Faculty of Medicine, Cairo University, Cairo 11562; Departments of \\ ${ }^{2}$ Clinical Pathology and ${ }^{3}$ Pediatrics, Faculty of Medicine, Zagazig University, Zagazig 44111, Egypt
}

Received October 9, 2014; Accepted November 6, 2014

DOI: $10.3892 / \mathrm{mco} .2014 .466$

\begin{abstract}
Similar to autoimmune diseases, there are clear associations between resistance or susceptibility to cancer and the classic human leukocyte antigen (HLA) profile of an individual. HLA-associated susceptibility to childhood acute lymphoblastic leukemia (ALL) may provide clues to leukemogenesis in general and to the role of other risk factors. The present study aimed to determine the association between the HLA-DRB1 genotype and susceptibility to ALL in children and to assess the prognostic value of HLA-DRB1 alleles in these patients. This study included 50 ALL patients who were consecutively admitted to the Pediatric Oncology Unit of Zagazig University Hospital and 50 gender-matched healthy volunteers as a control group. The patients were subjected to full clinical history, thorough clinical examination and routine laboratory investigations. Molecular HLA-DRB1 typing for patients and controls using the reverse sequence-specific oligonucleotide probe technique was performed. HLA-DRB1*04 allele frequency was significantly higher in female patients compared to that in female controls $(\mathrm{P}=0.03)$ and in patients aged $<10$ years compared to those aged $\geq 10$ years at the time of diagnosis $(\mathrm{P}=0.01)$. HLA-DRB1*11 allele frequency was significantly higher in high-risk compared to standard-risk patients $(\mathrm{P}=0.01)$ and in refractory patients compared to those who achieved remission $(\mathrm{P}=0.02)$. In conclusion, the HLA-DRB1*04 allele appears to be a female-specific susceptibility factor for the acquisition of childhood ALL and it may affect the age of onset of ALL. In addition, the HLA-DRB1*11 allele may be of prognostic significance in childhood ALL. However, further larger studies are required to support the conclusions drawn from this study.
\end{abstract}

Correspondence to: Dr Tamer H. Hassan, Department of Pediatrics, Zagazig University, Zagazig University Street, Zagazig 44111, Egypt

E-mail: dr.tamerhassan@yahoo.com

Key words: human leukocyte antigen-DRB1, acute lymphoblastic leukemia, polymorphism, childhood

\section{Introduction}

Acute lymphoblastic leukemia (ALL) is the most common malignancy diagnosed in children, representing one quarter of all pediatric cancers worldwide (1).

Over 40 years since the first associations between particular human leukocyte antigen (HLA) profiles and disease susceptibility were described, the identification of large numbers of HLA-associated diseases parallels our improved understanding of the genetic complexity of the HLA system and its extensive polymorphism. These associations involve malignant as well as autoimmune diseases, including the well-known association between HLA-B27 and ankylosing spondylitis. Over time, several hundred diseases have been reported to occur more frequently in individuals with particular HLA genotypes (2). These diseases include a broad spectrum of immune-mediated diseases involving all major organ systems, certain malignancies, infectious diseases and, more recently, adverse reactions to particular drugs (2).

One of the factors that may determine whether a child develops ALL is how it responds to the candidate infection. Since immune responses to infection are under the partial control of HLA genes, an association between an HLA allele and ALL may provide support for an infectious etiology (3).

The biological significance of the association between HLA types and acute leukemia is emphasized by the fact that HLA may be involved in disease susceptibility or resistance. It is important to investigate the HLA-associated susceptibility to childhood ALL, as this may provide clues to leukemogenesis in general and to the role of other risk factors (4).

The present study aimed to determine the association between the HLA-DRB1 genotype and the susceptibility to ALL in children and to evaluate the prognostic value of HLA-DRB1 alleles in these patients.

\section{Patients and methods}

Patients. This study was performed on 50 ALL patients who were consecutively admitted to the Pediatric Oncology Unit of Zagazig University Hospital and 50 gender-matched healthy volunteers as a control group.

The patients were subjected to: i) full clinical history and thorough clinical examination; and ii) routine laboratory 
investigations, including complete blood count, examination of Leishman-stained peripheral blood smear, bone marrow aspiration with examination of Leishman- and peroxidase-stained films and immunophenotyping by flow cytometry.

Molecular HLA-DRBI typing and risk classification. The patients and controls were subjected to molecular HLA-DRB1 typing and the patients also underwent risk classification.

HLA typing. EDTA-anticoagulated whole-blood samples were collected from patients and controls and processed as follows: i) DNA extraction using the spin column technique (QIAamp DNA Blood Mini kits; Qiagen, Hilden, Germany) was performed according to the manufacturer's guidelines; ii) polymerase chain reaction (PCR) amplification of the HLA-DRB1 gene (exon 2) target (INNO-LiPA HLA-DRB1 Amplification Plus kit; Fujirebio Europe, Ghent, Belgium) was performed and the amplicons were chemically denatured to form single-stranded DNA. Reverse dot-blotting was performed on a nylon membrane (INNO-LiPA HLA-DRB1 Plus strips, Fujirebio Europe), which contains an array of immobilized, sequence-specific oligonucleotide (SSO) probes. The biotin-labeled amplicons were then bound (hybridized) to these SSO probes, which contain a complementary target sequence and, thus, were captured onto the membrane strip; iii) visualization of the results was achieved by incubating with an enzyme conjugate (streptavidin and alkaline phosphatase), which binds to the biotin of the PCR product, followed by the addition of a substrate. The bands with the captured PCR product turned blue. Interpretation was achieved by entering the band pattern into a computer program.

Risk classification. The patients were divided into standard- and high-risk groups according to the Children's Cancer Group (CCG) risk stratification (5). Standard-risk patients were defined as follows: age 1-9 years; initial total leukocyte count $<50,000 / \mathrm{mm}^{3}$; precursor B-ALL immunophenotype; and no central nervous system (CNS) manifestations or testicular infiltration. High-risk patients exhibited any of the following at diagnosis: age $\geq 10$ years; initial total leukocyte count $\geq 50,000 / \mathrm{mm}^{3}$; T-ALL immunophenotype; CNS manifestations; and overt testicular leukemia.

Children with mature B-ALL were considered to be among the high-risk patients and were treated separately using the LMB-96 protocol, which is an international protocol conducted by three cooperative groups (International Society for Pediatric Oncology, CCG and United Kingdom Children's Cancer Study Group) and (6).

Statistical analysis. The data were checked, entered and analyzed using Epi Info 6 statistical software (Centers for Disease Control and Prevention, Atlanta, GA, USA). The results are expressed as mean \pm standard deviation for quantitative variables and as number and percentage for qualitative variables. The differences in the frequencies of HLA-DRB1 alleles among the investigated groups were analyzed using the Chi-square test with Yates' correction and the two-tailed Fisher's exact test for results $\leq 5$. Each allele frequency in the patients was compared against the same allele in controls. The odds ratios (ORs) with 95\% confidence intervals were calculated. $\mathrm{P} \leq 0.05$ was considered to indicate statistically significant differences.
Table I. HLA-DRB1 allele frequencies in patients and controls.

\begin{tabular}{lcccc}
\hline $\begin{array}{l}\text { HLA- } \\
\text { DRB1 } \\
\text { alleles }\end{array}$ & $\begin{array}{c}\text { Patient } \\
\text { no. }(\%) \\
(\mathrm{n}=50)^{\mathrm{a}}\end{array}$ & $\begin{array}{c}\text { Control } \\
\text { no. }(\%) \\
(\mathrm{n}=50)^{\mathrm{a}}\end{array}$ & OR (95\% CI) & P-value \\
\hline$* 01$ & $2(4.0)$ & $5(10.0)$ & $0.38(0.05-2.35)$ & 0.43 \\
$* 03$ & $10(20.0)$ & $10(20.0)$ & $1(0.34-2.96)$ & 0.80 \\
$* 04$ & $21(42.0)$ & $12(24.0)$ & $2.29(0.9-5.93)$ & 0.13 \\
$* 07$ & $13(26.0)$ & $7(14.0)$ & $2.16(0.71-6.76)$ & 0.21 \\
$* 08$ & $2(4.0)$ & $3(6.0)$ & $0.65(0.07-5.11)$ & 0.99 \\
$* 09$ & $0(0.0)$ & $3(6.0)$ & $0(0.0-2.23)$ & 0.24 \\
$* 10$ & $4(8.0)$ & $4(8.0)$ & $1(0.19-5.14)$ & 1.00 \\
$* 11$ & $15(30.0)$ & $14(28.0)$ & $1.1(0.43-2.86)$ & 1.00 \\
$* 12$ & $0(0.0)$ & $3(6.0)$ & $0(0.0-2.23)$ & 0.24 \\
$* 13$ & $14(28.0)$ & $19(38.0)$ & $0.63(0.25-1.6)$ & 0.39 \\
$* 14$ & $3(6.0)$ & $4(8.0)$ & $0.73(0.12-4.18)$ & 0.99 \\
$* 15$ & $6(12.0)$ & $9(18.0)$ & $0.62(0.18-2.13)$ & 0.57 \\
$* 16$ & $2(4.0)$ & $2(4.0)$ & $1(0.1-10.47)$ & 1.00 \\
\hline
\end{tabular}

${ }^{\mathrm{a}} \mathrm{A}$ total of 8 patients and 5 controls were homozygous. HLA, human leukocyte antigen; OR, odds ratio; $\mathrm{CI}$, confidence interval.

Ethics. This study was conducted in accordance with the ethical standards of the Helsinki Declaration of 1964, as revised in 2000 (7) and was approved by the Institutional Review Board of the Faculty of Medicine, Zagazig University. Informed consent was obtained from all the study participants.

\section{Results}

Patient characteristics. The mean age of the patients at diagnosis was $4.9 \pm 3.3$ years (range, 2-13 years). The patients included 32 males and 18 females, with a male:female ratio of 1.8:1. The most frequent clinical manifestation was pallor (88\%), followed by fever (74\%), hepatosplenomegaly (72\%), lymphadenopathy (54\%), purpura (40\%) and bone pain (30\%). CNS was involved in only $4 \%$ of the patients. A total of $72 \%$ of the patients had a total leukocyte count $<50,000 / \mathrm{mm}^{3}$ and $28 \%$ had a total leukocyte count $\geq 50,000 / \mathrm{mm}^{3}$. A total of $88 \%$ of the patients had precursor B-ALL, 10\% had T-ALL and $2 \%$ had mature B-ALL.

Allele frequency determination. HLA-DRB1*04, *11, *13, *07 and $* 03$ were the most frequent alleles in the patients $(42,30,28$, 26 and $20 \%$, respectively). HLA-DRB1*09 and HLA-DRB1*12 alleles were not detected in any patients. HLA-DRB1*13, *11, $* 04, * 03$ and $* 15$ were the most frequent alleles in the controls $(38,28,24,20$ and $18 \%$, respectively). There was no significant difference between patients and controls as regards the frequencies of different HLA-DRB1 alleles $(\mathrm{P}>0.05)$ (Table I).

A total of 8 patients were homozygous for HLA-DRB1, namely 6 males (homozygous HLA-DRB $1 * 03, * 04, * 04, * 07$, $* 13$ and *14) and 2 females (homozygous HLA-DRB1*11 and *13); in addition, 5 controls were homozygous for HLA-DRB1, namely 2 males (HLA-DRB $1 * 04$ and $* 11$ ) and 3 females (HLA-DRB1*03, *04 and *04). 
Table II. HLA-DRB1 allele frequencies in female patients and female controls.

\begin{tabular}{lcccc}
\hline HLA- & $\begin{array}{c}\text { Female } \\
\text { patients, } \\
\text { no. }(\%) \\
(\mathrm{n}=18)\end{array}$ & $\begin{array}{c}\text { Female } \\
\text { controls, } \\
\text { no. }(\%) \\
(\mathrm{n}=18)\end{array}$ & OR $(95 \% \mathrm{CI})$ & P-value \\
alleles & (1) & 0.0 & 0.95 \\
\hline *1 & $0(0.0)$ & $1(5.6)$ & $0.63(0.06-5.64)$ & 0.99 \\
$* 03$ & $2(11.1)$ & $3(16.7)$ & 0.03 \\
$* 04$ & $10(55.6)$ & $3(16.7)$ & $6.25(1.09-40.4)$ & 0.03 \\
$* 07$ & $6(33.3)$ & $3(16.7)$ & $2.5(0.42-16.32)$ & 0.44 \\
$* 08$ & $1(5.6)$ & $1(5.6)$ & $1(0.0-40.56)$ & 1.00 \\
$* 09$ & $0(0.0)$ & $1(5.6)$ & 0.0 & 0.99 \\
$* 10$ & $0(0.0)$ & $1(5.6)$ & 0.0 & 0.99 \\
$* 11$ & $6(33.3)$ & $4(22.2)$ & $1.75(0.32-9.91)$ & 0.70 \\
$* 12$ & $0(0.0)$ & $2(11.1)$ & 0.0 & 0.48 \\
$* 13$ & $5(27.8)$ & $7(38.9)$ & $0.6(0.12-3.01)$ & 0.71 \\
$* 14$ & $0(0.0)$ & $2(11.1)$ & 0.0 & 0.48 \\
$* 15$ & $3(16.7)$ & $4(22.2)$ & $0.7(0.1-4.74)$ & 0.99 \\
$* 16$ & $1(5.6)$ & $1(5.6)$ & $1(0.0-40.56)$ & 1.00 \\
\hline
\end{tabular}

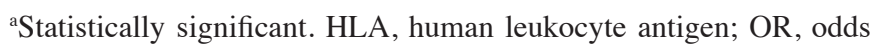
ratio; $\mathrm{CI}$, confidence interval.

There was no significant difference between male patients and male controls as regards the frequencies of different HLA-DRB1 alleles $(\mathrm{P}>0.05)$. However, the HLA-DRB $1 * 04$ allele frequency was significantly higher among female patients compared to that among female controls (55.6 vs. 16.7\%, respectively; $\mathrm{P}=0.03$ ) (Table II).

Based on the CCG risk classification, the ALL patients were divided regarding their age at diagnosis into two groups, namely those aged $<10$ and those aged $\geq 10$ years. The HLA-DBR $1 * 04$ allele frequency was significantly higher among patients aged $<10$ years compared to those aged $\geq 10$ years at diagnosis ( 50.0 vs. $0.0 \%$; $\mathrm{P}=0.01$ ) (Table III).

There was no significant difference among patients with different immunophenotypes as regards the frequencies of different HLA-DRB1 alleles $(\mathrm{P}>0.05)$.

The HLA-DRB1*11 allele frequency was significantly higher in high-risk compared to standard-risk patients (50.0 vs. $14.3 \%$; $\mathrm{P}=0.01$ ) (Table IV).

Of the 50 included patients, 10 were referred to other hospitals prior to treatment initiation and 10 succumbed to the disease; 1 patient with mature B-ALL succumbed to acute renal failure prior to treatment initiation, 7 patients died during treatment due to chemotherapy-related toxicity and infections and 2 patients died following disease relapse.

Following exclusion of the 10 referred patients and the 1 patient who died prior to treatment initiation, the remaining 39 patients were divided into the remission $(\mathrm{n}=34)$ and refractory $(\mathrm{n}=5)$ groups. The HLA-DR $1 * 11$ allele frequency was significantly higher among refractory patients compared to those who achieved remission ( 80.0 vs. $23.5 \%$; $\mathrm{P}=0.02$ ) (Table V).

Following exclusion of the 10 referred patients and the 8 patients who died during the study due to causes other
Table III. HLA-DRB1 allele frequencies in relation to patient age at diagnosis.

\begin{tabular}{|c|c|c|c|c|}
\hline \multirow[b]{2}{*}{$\begin{array}{l}\text { HLA- } \\
\text { DRB1 } \\
\text { alleles }\end{array}$} & \multicolumn{2}{|c|}{ Patient age } & \multirow[b]{2}{*}{ OR $(95 \%$ CI $)$} & \multirow[b]{2}{*}{ P-value } \\
\hline & $\begin{array}{c}<10 \text { yrs, } \\
\text { no. }(\%) \\
(n=42)\end{array}$ & $\begin{array}{c}\geq 10 \text { yrs } \\
\text { no. }(\%) \\
(n=8)\end{array}$ & & \\
\hline$* 01$ & $2(4.8)$ & $0(0.0)$ & 0.0 & 1.00 \\
\hline$* 03$ & $6(14.3)$ & $4(50)$ & $0.16(0.03-0.85)$ & 0.04 \\
\hline$* 04$ & $21(50.0)$ & $0(0.0)$ & 0.0 & $0.01^{\mathrm{a}}$ \\
\hline$* 07$ & $10(23.8)$ & $3(37.5)$ & $0.52(0.10-2.57)$ & 0.66 \\
\hline$* 08$ & $1(2.3)$ & $1(12.5)$ & $0.17(0-3.05)$ & 1.00 \\
\hline$* 10$ & $4(9.5)$ & $0(0.0)$ & 0.0 & 0.60 \\
\hline$* 11$ & $12(28.6)$ & $3(37.5)$ & $0.66(0.13-3.2)$ & 0.68 \\
\hline$* 13$ & $12(28.6)$ & $2(25)$ & $1.2(0.21-6.80)$ & 1.00 \\
\hline$* 14$ & $3(7.1)$ & $0(0.0)$ & 0.0 & 1.00 \\
\hline$* 15$ & $5(11.9)$ & $1(12.5)$ & $0.94(0.09-9.3)$ & 0.65 \\
\hline$* 16$ & $1(2.3)$ & $1(12.5)$ & $0.17(0-3.05)$ & 1.00 \\
\hline
\end{tabular}

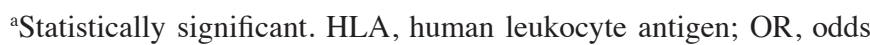
ratio; CI, confidence interval.

Table IV. HLA-DRB1 allele frequencies in relation to risk.

\begin{tabular}{|c|c|c|c|c|}
\hline \multirow[b]{2}{*}{$\begin{array}{l}\text { HLA- } \\
\text { DRB1 } \\
\text { alleles }\end{array}$} & \multicolumn{2}{|c|}{ Risk } & \multirow[b]{2}{*}{ OR $(95 \% \mathrm{CI})$} & \multirow[b]{2}{*}{$\mathrm{P}$-value } \\
\hline & $\begin{array}{c}\text { Standard, } \\
\text { no. }(\%) \\
(\mathrm{n}=28)\end{array}$ & $\begin{array}{c}\text { High, } \\
\text { no. }(\%) \\
(\mathrm{n}=22)\end{array}$ & & \\
\hline$* 01$ & $1(3.6)$ & $1(4.5)$ & $0.78(0.02-30.61)$ & 1.00 \\
\hline$* 03$ & $5(17.9)$ & $5(22.7)$ & $0.74(0.15-3.61)$ & 0.73 \\
\hline$* 04$ & $13(46.4)$ & $8(36.4)$ & $1.52(0.42-5.58)$ & 0.67 \\
\hline$* 07$ & $7(25.0)$ & $6(27.3)$ & $0.89(0.21-3.78)$ & 0.88 \\
\hline$* 08$ & $1(3.6)$ & $1(4.5)$ & $0.78(0.02-30.61)$ & 1.00 \\
\hline$* 10$ & $3(10.7)$ & $1(4.5)$ & $2.52(0.2-67.98)$ & 0.62 \\
\hline$* 11$ & $4(14.3)$ & $11(50.0)$ & $0.17(0.03-0.75)$ & $0.01^{\mathrm{a}}$ \\
\hline$* 13$ & $10(35.7)$ & $4(18.2)$ & $2.5(0.56-11.75)$ & 0.29 \\
\hline$* 14$ & $3(10.7)$ & $0(0.0)$ & 0.0 & 0.24 \\
\hline$* 15$ & $3(10.7)$ & $3(13.6)$ & $0.76(0.11-5.47)$ & 1.00 \\
\hline$* 16$ & $1(3.6)$ & $1(4.5)$ & $0.78(0.02-30.61)$ & 1.00 \\
\hline
\end{tabular}

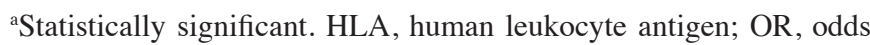
ratio; CI, confidence interval.

than relapse, the remaining 32 patients were divided into the relapsed $(n=4)$ and non-relapsed groups $(n=28)$. There was no significant difference between the two groups as regards the frequencies of different HLA-DRB1 alleles $(\mathrm{P}>0.05)$.

Following exclusion of the 10 referred patients, the remaining 40 patients were divided into the survivors $(n=30)$ and those who succumbed to the disease $(n=10)$. There was no significant difference between the two groups as regards the frequencies of different HLA-DRB1 alleles $(\mathrm{P}>0.05)$. 
Table V. HLA-DRB1 allele frequencies in relation to induction of remission.

\begin{tabular}{lcccc}
\hline HLA- & $\begin{array}{c}\text { Remission } \\
\text { group, } \\
\text { DRB1 } \\
\text { no. }(\%) \\
(\mathrm{n}=34)\end{array}$ & $\begin{array}{c}\text { Refractory } \\
\text { group, } \\
\text { no. }(\%) \\
(\mathrm{n}=5)\end{array}$ & OR (95\% CI) & P-value \\
\hline$* 01$ & $1(2.9)$ & $1(20.0)$ & $0.12(0.0-2.3)$ & 0.24 \\
$* 03$ & $6(17.6)$ & $2(40.0)$ & $0.32(00-2.3)$ & 0.56 \\
$* 04$ & $15(44.1)$ & $0(0.0)$ & 0.0 & 0.13 \\
$* 07$ & $9(26.4)$ & $1(20.0)$ & $1.4(0.14-14.6)$ & 1.00 \\
$* 08$ & $1(2.9)$ & $0(0.0)$ & 0.0 & 1.00 \\
$* 10$ & $3(8.8)$ & $1(20.0)$ & $0.38(0.0-4.6)$ & 0.99 \\
$* 11$ & $8(23.5)$ & $4(80.0)$ & $0.07(0.0-0.79)$ & $0.02^{\mathrm{a}}$ \\
$* 13$ & $10(29.4)$ & $1(20.0)$ & $1.6(0.16-16.0)$ & 1.00 \\
$* 14$ & $2(5.8)$ & $0(0.0)$ & 0.0 & 1.00 \\
$* 15$ & $6(17.6)$ & $0(0.0)$ & 0.0 & 0.57 \\
\hline
\end{tabular}

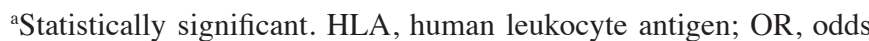
ratio; $\mathrm{CI}$, confidence interval.

\section{Discussion}

Genetic and environmental factors play an interactive role in the development of childhood ALL. Since the demonstration of a major histocompatibility complex effect on mouse leukemia in 1964, an HLA association has been considered as a possible genetic risk factor (8).

In the present study, there was no significant difference between patients and controls as regards the frequencies of different HLA-DRB1 alleles ( $>>0.05)$. In addition, there was no significant difference between male patients and male controls as regards the frequencies of different HLA-DRB1 alleles $(\mathrm{P}>0.05)$. However, the HLA-DRB1*04 allele frequency was significantly higher among female patients compared to female controls (55.6 vs. $16.7 \% ; \mathrm{P}=0.03$ ), indicating that HLA-DRB1*4 is a female-specific susceptibility allele for childhood ALL.

The putative role of the HLA class-II DR antigen in ALL risk was first suggested in the late 1970s by de Moerloose et al (9), who demonstrated an association with DR7. Additional evidence specifically regarding childhood ALL was available shortly after the studies conducted by von Fliedner et al, one of which confirmed the DR7 association (10), whereas the other revealed a higher sharing of DR antigens among parents of children with leukemia than expected (11).

By contrast, Dorak et al (8) reported that the frequency of the HLA-DRB1*04 allele was higher $(\mathrm{P}=0.0005, \mathrm{OR}=2.9)$, whereas the frequency of the HLA-DRB $1 * 13$ allele was lower in male patients.

In 2002, a study that was performed on an Indian population demonstrated that the HLA-DRB1*04 and HLA-DRB1*13 alleles are susceptible and protective alleles, respectively, in ALL patients (12).

In their study on 106 Iranian patients with ALL, Yari et al (13) concluded that HLA-DRB1*13, which exhibited a decrease among the patients, appeared to be protective against ALL, whereas HLA-DRB $1 * 04$, which was moderately increased among the patients, was considered a susceptibility allele for childhood ALL.

A significant increase in the frequency of the HLA-DRB1*04 allele in the overall and male ALL patients was reported in a Turkish study (4). In addition, a significantly lower frequency of the HLA-DRB1*13 allele was observed among female patients compared to female controls (4).

The association between HLA class II alleles and ALL was investigated in 20 Iranian patients with leukemia (14). The results revealed a significant increase in the frequency of the HLA-DRB1*04 allele in ALL patients compared to that in controls.

DRB1*15, a risk allele for multiple sclerosis, has also been implicated in the susceptibility to childhood ALL. At least two studies have reported such an association; one was conducted in a Chinese population comprising 162 childhood ALL cases and 1,000 controls (15), whereas the other was conducted in a UK population, investigated the effects by gender and observed an association only in females, which is consistent with a female-specific effect of the allele DRB $1 * 15: 01$, also observed in multiple sclerosis (16).

The discrepancy between the results of different studies, including our results, may be attributed to the differences in population race and geographical distribution.

Studies investigating the pathogenic effects of a gene should include factors such as the differences in the frequency distributions of the gene between the patient and the control groups (the stronger the effect of a gene, the more significant the differences in frequency distribution) and early or late age at disease onset in gene carriers (the stronger the effects, the earlier the onset) (17).

In our study, the HLA-DBR $1 * 04$ allele frequency was significantly higher in patients aged $<10$ years compared to those aged $\geq 10$ years at the time of diagnosis (50.0 vs. $0.0 \%$; $\mathrm{P}=0.01$ ). From these results, it was suggested that the HLA-DRB1 allele may affect the age at onset in ALL.

To the best of our knowledge, there are no studies in the literature associating the HLA-DRB1 allele frequencies with the age at onset in ALL. However, this allele has been investigated in relation to other cancers in adults $(17,18)$.

The mean age of patients with colorectal cancer who carry the HLA-DQB1*02 allele was found to be lower compared to that of subjects without the allele $(\mathrm{P}<0.05)$, suggesting that this allele is associated with colorectal cancer susceptibility (17). In another study, the frequency of HLA alleles in 50 chronic myeloid leukemia patients in relation to age at onset was investigated; the authors concluded that the frequency of the HLA-DRB1*07 $(\mathrm{P}=0.03)$ and DQA1*0201 $(\mathrm{P}=0.03)$ alleles was higher among patients aged $<35$ years (18).

Our results demonstrated that the HLA-DRB1*11 allele frequency was significantly higher among high-risk compared to standard-risk patients (50.0 vs. $14.3 \% ; \mathrm{P}=0.01)$ and in refractory patients compared to those who achieved remission ( 80.0 vs. $23.5 \% ; \mathrm{P}=0.02)$. These results indicated that the HLA-DRB1*11 allele may be a predictor for prognosis and treatment outcome in children with ALL. However, there was no significant difference between the relapsed and non-relapsed groups and between survivors and those who succumbed to the disease as regards the frequencies of different HLA-DRB1 alleles $(\mathrm{P}>0.05)$. 
The significance of the HLA alleles as prognostic indicators in childhood leukemia has been investigated in a limited number of studies. Casper et al (19) reported an increased incidence of relapse associated with HLA-DR5, with a higher rate of disease-free remission associated with DR7; however, Takeuchi et al (20) and Lauten et al (21) did not identify an association with outcome in childhood leukemia.

In conclusion, the HLA-DRB1*04 allele appears to be a female-specific susceptibility factor for the development of childhood ALL and it may affect the age at onset for ALL. In addition, the HLA-DRB1*11 allele may be of prognostic value in childhood ALL. However, further larger studies are required to support our findings.

\section{References}

1. Ribera JM and Oriol A: Acute lymphoblastic leukemia in adolescents and young adults. Hematol Oncol Clin North Am 23: 1033-1042, 2009.

2. Howell WM: HLA and disease: guilt by association. Int $\mathrm{J}$ Immunogenet 41: 1-12, 2014.

3. Dearden SP, Taylor GM, Gokhale DA, et al: Molecular analysis of HLA-DQB1 alleles in childhood common acute lymphoblastic leukaemia. Br J Cancer 73: 603-609, 1996.

4. Ozdilli K, Oguz FS, Anak S, Kekik C, Carin M and Gedikoglu G: The frequency of HLA class I and II alleles in Turkish childhood acute leukaemia patients. J Int Med Res 38: 1835-1844, 2010.

5. Pui CH, Carroll WL, Meshinchi S and Arceci RJ: Biology, risk stratification, and therapy of pediatric acute leukemias: an update. J Clin Oncol 29: 551-565, 2011.

6. Cairo MS, Gerrard M and Patte C: A new protocol for treatment of mature B-cell lymphoma/leukemia (BCLL): FAB LMB 96, a SFOP LMB 96/CCG-5961/UKCCSG NHL 9600 international cooperative study. Med Pediatr Oncol 29: 357A, 1997.

7. Christie B: Doctors revise declaration of Helsinki. BMJ 321: 913 , 2000.

8. Dorak MT, Lawson T, Machulla HK, Darke C, Mills KI and Burnett AK: Unravelling an HLA-DR association in childhood acute lymphoblastic leukemia. Blood 94: 694-700, 1999.

9. de Moerloose P, Chardonnens X, Vassalli P and Jeannet M: HL-A $\mathrm{D}$ antigens from B-lymphocytes and susceptibility to certain diseases. Schweiz Med Wochenschr 107: 1461, 1977 (In French).
10. von Fliedner VE, Sultan-Khan Z and Jeannet M: HLA-DRw antigens associated with acute leukemia. Tissue Antigens 16: 399-404, 1980

11. von Fliedner VE, Merica H, Jeannet M, et al: Evidence for HLA-linked susceptibility factors in childhood leukemia. Hum Immunol 8: 183-193, 1983.

12. Jaini R, Kaur G and Mehra NK: Heterogeneity of HLA-DRB1*04 and its associated haplotypes in the North Indian population. Hum Immunol 63: 24-29, 2002.

13. Yari F, Sobhani M, Sabaghi F, Zaman-Vaziri M, Bagheri N and Talebian A: Frequencies of HLA-DRB1 in Iranian normal population and in patients with acute lymphoblastic leukemia. Arch Med Res 39: 205-208, 2008.

14. Atash beik SM, Akbari MT, Sayad A, et al: The association of HLA-DRB1 gene polymorphisms with acute lymphoblastic leukemia (ALL) in Iranian patients. J Biol Today's World 2: 173-178, 2013.

15. Wang XJ, Ai XF, Sun HY, et al: Relation of HLA-DRB $1 * 15$ with pathogensis in 162 childhood cases of acute lymphoblastic leukemia. Chin Assoc Pathophysiol 17: 1507-1510, 2009 (In Chinese).

16. Morrison BA, Ucisik-Akkaya E, Flores H, Alaez C, Gorodezky C and Dorak MT: Multiple sclerosis risk markers in HLA-DRA, HLA-C, and IFNG genes are associated with sex-specific childhood leukemia risk. Autoimmunity 43: 690-697, 2010.

17. Tong F, Yu W and Liu H: Efficient association analysis between colorectal cancer and allelic polymorphisms of HLA-DQB1 by comparison of age of onset. Oncol Lett 3: 517-519, 2012

18. Amirzargar AA, Khosravi F, Dianat SS, et al: Association of HLA class II allele and haplotype frequencies with chronic myelogenous leukemia and age-at-onset of the disease. Pathol Oncol Res 13: 47-51, 2007.

19. Casper JT, Marrari M, Piaskowski V, Lauer SJ and Duquesnoy RJ: Association between HLA-D region antigens and disease-free survival in childhood non-T, non-B acute lymphocytic leukemia. Blood 60: 698-702, 1982.

20. Takeuchi S, Takeuchi N, Tsukasaki K, et al: Genetic polymorphisms in the tumor necrosis factor locus in childhood acute lymphoblastic leukaemia. Br J Haematol 119: 985-987, 2002.

21. Lauten M, Matthias T, Stanulla M, Beger C, Welte K and Schrappe M: Association of initial response to prednisone treatment in childhood acute lymphoblastic leukaemia and polymorphisms within the tumor necrosis factor and interleukin-10 genes. Leukemia 16: 1437-1442, 2002. 